\title{
MicroRNA-217 inhibits cell proliferation, invasion and migration by targeting Tpd5212 in human pancreatic adenocarcinoma
}

\author{
QIUYANG CHEN ${ }^{1,2}$, PENG WANG $^{1,2}$, YUE FU $^{1,2}$, XINCHUN LIU $^{1,2}$, WENBIN XU $^{1,2}$, JISHU WEI $^{1,2}$, \\ WENTAO GAO $^{1,2}$, KUIRONG JIANG ${ }^{1,2}$, JUNLI WU ${ }^{1,2}$ and YI MIAO ${ }^{1,2}$ \\ ${ }^{1}$ Pancreas Center, The First Affiliated Hospital of Nanjing Medical University; \\ ${ }^{2}$ Pancreas Institute, Nanjing Medical University, Nanjing, Jiangsu 210029, P.R. China
}

Received March 8, 2017; Accepted August 10, 2017

DOI: $10.3892 /$ or.2017.6036

\begin{abstract}
MicroRNAs (miRNAs) play important roles in the regulation of various tumor biological processes including proliferation and apoptosis. miR-217 has been implicated in many types of cancer, whereas its expression and potential biological function in human pancreatic adenocarcinoma (HPAC) remain unclear. We aimed to investigate the clinical significance of miR-217 in patients with pancreatic carcinoma and its role and underlying molecular mechanism in HPAC. We collected 15 pairs of pancreatic cancer and normal pancreas tissues to evaluate the expression of miR-217 and tumor protein D52-like 2 (Tpd5212). Then, we transfected AsPC-1 cells with miR-217 mimics or Tpd5212 siRNA to detect the effect on cell proliferation, apoptosis, invasion, migration and the cell cycle. In addition, miR-217 mimics and Tpd5212 expression plasmids were co-transfected into AsPC-1 cells to further investigate the mechanism of miR-217 and Tpd5212 in HPAC tumorigenesis. Finally, exploration of related signaling pathways was carried out. Herein, we found that the expression of miR-217 was significantly downregulated in HPAC tissues as compared with that observed in adjacent normal tissues. Further functional assays showed that restoration of the expression of miR-217 inhibited cell proliferation, invasion and migration, induced apoptosis, and caused cell cycle arrest of HPAC cells. Notably, Tpd5212 was identified as a functional target of miR-217 in HPAC. Furthermore, an inverse correlation between miR-217 and Tpd5212 expression was observed in the HPAC tissues. Downregulation of Tpd5212 had an effect similar to that following overexpression of miR-217, and upregulation of Tpd5212 reversed the effects of the overexpression of miR-217. Finally, we found that overexpression of miR-217 or knockdown of Tpd5212 suppressed
\end{abstract}

Correspondence to: Dr Junli Wu or Dr Yi Miao, Pancreas Center, The First Affiliated Hospital of Nanjing Medical University, 300 Guangzhou Road, Nanjing, Jiangsu 210029, P.R. China

E-mail: junliwu1973@hotmail.com

E-mail: miaoyi@njmu.edu.cn

Key words: human pancreatic adenocarcinoma, miR-217, proliferation, migration, invasion the PIK3CA/AKT signaling pathways. In addition, this may explain the effect of miR-217/Tpd5212 on HPAC development. Taken together, these results suggest a critical role of miR-217 in suppressing proliferation, migration and invasion of HPAC cells by targeting Tpd5212. Targeting the miR-217/Tpd5212 axis may be a new therapeutic application with which to treat patients with HPAC in the future.

\section{Introduction}

Human pancreatic adenocarcinoma (HPAC) is recognized as one of the most fatal malignant neoplasms. Although therapeutic management has been improved using a variety of treatment approaches, the 5-year survival rate of patients with HPAC remains $7.7 \%$, making HPAC a cancer with one of the worst prognoses of any major malignancy $(1,2)$. Thus, it is quite urgent to understand the molecular mechanisms by which HPAC initiates, progresses, invades, and recurs to develop novel and effective therapeutic strategies for HPAC.

MicroRNAs (miRNAs) are 18-25 nucleotide-long, singlestranded, non-coding RNA molecules that negatively regulate gene expression by binding to the regions of sequence complementarity to the 3 ' untranslated region ('UTR) of mRNAs, leading to either the degradation or translational inhibition of mRNAs (3). Previous research has found that miRNAs are involved in various cellular processes, including cell proliferation, apoptosis, migration and invasion, the cell cycle and stem cell renewal $(4,5)$. In addition, evidence suggests that miRNAs are aberrantly expressed in various cancers, and they play oncogenic or tumor-suppressive roles in these cancers (6-8). Differentially expressed miRNAs may also offer novel therapeutic approaches for the more successful treatment of $\operatorname{HPAC}(9,10)$.

As a member of the miRNA family, microRNA-217 (miR-217) has been shown to be involved in the initiation and development of many human types of cancers, and functions as a tumor suppressor in the majority of cancers, such as ovarian cancer, osteosarcoma, esophageal squamous cell carcinoma, hepatocellular carcinoma, pancreatic ductal adenocarcinoma and lung and colorectal cancer (11-17). However, the clinical significance of miR-217 in pancreatic carcinoma and its underlying molecular pathways involved in pancreatic carcinoma have not been investigated. 
Tumor protein D52-like 2, known as hD54 in previous studies (Tpd5212), is a member of the TPD52 family which has been implicated in multiple human cancers (18-20). Further research has demonstrated that the TPD52 gene encodes regulators of cancer cell proliferation, indicating that TPD52 may be important for maintaining tumorigenesis and metastasis of cancer cells (21). However, its direct effect and molecular mechanism involved in HPAC development have not yet been elucidated.

In the present study, we investigated the expression and role of miR-217 in human HPAC. The results indicated that miR-217 functions as a tumor suppressor in HPAC. In addition, overexpression of Tpd5212 reversed the effects of miR-217 restoration in AsPC-1 cells. All these results suggest that miR-217 exerts a tumor-suppressor role in HPAC, at least in part, by repressing Tpd5212 expression. The miR-217/Tpd5212 axis may be a new therapeutic strategy with which to treat patients with HPAC in the future.

\section{Materials and methods}

Tissues and cell lines. Thirty samples of pancreatic carcinoma and para-carcinoma tissues were collected from The First Affiliated Hospital of Nanjing Medical University. The pancreatic carcinoma and normal tissues were snap-frozen in liquid nitrogen immediately after resection and stored at $-80^{\circ} \mathrm{C}$ until use. The present study was approved by the Research Ethics Committee of The First Affiliated Hospital of Nanjing Medical University.

The AsPC-1 cell line used in the present study was purchased from the American Type Culture Collection (ATCC; Manassas, VA, USA). Cells were cultured according to ATCC recommendations: RPMI-1640 medium with $10 \%$ fetal bovine serum (FBS) (Gibco, Grand Island, NY, USA) and penicillin/ streptomycin (Sigma, St. Louis, MO, USA) supplement in a $37^{\circ} \mathrm{C}$ humidified incubator supplied with $95 \%$ air and $5 \% \mathrm{CO}_{2}$.

Antibodies. Commercially available antibodies were used for all immunoblotting and immunofluorescence studies. Anti-Tpd5212, anti-PIK3CA, anti-p-PIK3CA, anti-AKT1/2 and anti-p-AKT1/2 were obtained from Abcam Co. (Cambridge, UK). Anti-GAPDH was obtained from Kangchen KangChen Bio-Tech (Shanghai, China). All secondary antibodies used were obtained from Boster (Beijing, China).

Cell transfection. miR-217 mimic (miR-217) and the corresponding negative control, the siRNAs targeting human Tpd5212c (si-Tpd5212) and the corresponding negative control were obtained form GenePharma Co., Ltd. (Shanghai, China). Tpd5212 overexpressing plasmids were construct using pCDNA3.1 (+) basic vectors in our laboratory. These molecular productions were transfected into AsPC-1 cells when cells were grown to 80-90\% confluence, using Lipofectamine 2000 (Invitrogen, Carlsbad, CA, USA) according to the manufacturer's instructions.

Quantitative real-time PCR. Tumor specimens and cells were subjected to total RNA extraction using TRIzol reagent (Takara, Tokyo, Japan). To detect RNA expression, complementary DNA (cDNA) was synthesized using a Bestar qPCR RT kit (DBI Bioscience, Ludwigshafen, Germany) according to the manufacturer's instructions. Amplification and detection of RNA were performed using a Bestar qPCR RT kit under the ABI 9700 PCR amplifier system (Applied Biosystems, Foster City, CA, USA). In detail, the primer sequences are as follows: for miR-150 detection, forward primer, ACA CTC CAG CTG GGT CTC CCA ACC CTT GTA CC and reverse primer, CTC AAC TGG TGT CGT GGA GTC GGC AAT TCA GTT GAG CAC TGG T; for miR-138 detection, forward primer, CTC AAC TGG TGT CGT GGA GTC GGC AAT TCA GTT GAG TCC AAT C and reverse primer, CTC AAC TGG TGT CGT GGA GTC GGC AAT TCA GTT GAG CGG CCT G; for miR-217 detection, forward primer, ACA CTC CAG CTG GGT ACT GCA TCA GGA ACT GA and reverse primer, CTC AAC TGG TGT CGT GGA GTC GGC AAT TCA GTT GAG TCC AAT C; for miR-205 detection, forward primer, ACA CTC CAG CTG GGT CCT TCA TTC CAC CGG AG and reverse primer, CTC AAC TGG TGT CGT GGA GTC GGC AAT TCA GTT GAG CAG ACT C; for miR-31 detection, forward primer, ACA CTC CAG CTG GGA GGC AAG ATG CTG GCA TA and reverse primer, CTC AAC TGG TGT CGT GGA GTC GGC AAT TCA GTT GAG AGC TAT G; for miR-34 detection, forward primer, ACA CTC CAG CTG GGT GGC AGT GTC TTA GCT GG and reverse primer, CTC AAC TGG TGT CGT GGA GTC GGC AAT TCA GTT GAG ACA ACC A; for Tpd5212 detection, forward primer, CATGACGTGCAGGTCTCTAGC and reverse primer, CATGACGTGCAGGTCTCTAGC. The miRNA and mRNA expression levels were normalized to those of GAPDH and U6, respectively, using the $2^{-\Delta \Delta \mathrm{Ct}}$ method.

Western blotting. Monolayer cells were grown to $80 \%$ confluence and then washed in ice-cold phosphate-buffered saline (PBS). Cells were lysed in Universal protein extraction buffer (Beyotime, Shanghai, China) applied with protease inhibitor phenylmethanesulfonyl fluoride (PMSF) (Genebase Gen-Tech Co., Ltd., Shanghai, China) $48 \mathrm{~h}$ after transfection. For western blotting, proteins were mixed with 5X SDS loading buffer [250 mM Tris- $\mathrm{HCl}$ (pH 6.8), 10\% SDS, $0.5 \%$ bromophenol blue, $50 \%$ glycerol, $5 \% \beta$-mercaptoethanol] and heated to $95^{\circ} \mathrm{C}$ for $8 \mathrm{~min}$ before separating with $4-12 \%$ SDS-PAGE. Proteins were then transferred to polyvinylidene difluoride membranes (Millipore, Billerica, MA, USA), and detected with appropriate primary antibodies and horseradish peroxidase-conjugated secondary antibodies. Finally, the membranes were visualized using chemiluminescent HRP substrate (Millipore). For Tpd5212 detection, anti-Tpd5212 antibody was at 1:2,000 dilution, and the Tpd5212 transfer membrane at a constant current $300 \mathrm{~mA}$ for $100 \mathrm{~min}$. For PIK3CA detection, the antiPIK3CA antibody was at 1:2,500 dilution, and the PIK3CA transfer membrane at a constant current $300 \mathrm{~mA}$ for $100 \mathrm{~min}$. For p-PIK3CA detection, the anti-P-PIK3CA antibody was at 1:800 dilution, and the p-PIK3CA transfer membrane at a constant current $300 \mathrm{~mA}$ for $100 \mathrm{~min}$. For AKT1/2 detection, the anti-AKT1/2 antibody was at 1:1,000 dilution, and the AKT1/2 transfer membrane at a constant current $300 \mathrm{~mA}$ for $50 \mathrm{~min}$. For p-AKT1/2 detection, the anti-P-AKT1/2 antibody was at 1:1,500 dilution, and the $\mathrm{p}-\mathrm{AKT} 1 / 2$ transfer membrane at a constant current $300 \mathrm{~mA}$ for $45 \mathrm{~min}$. For GAPDH detection, the anti-GAPDH antibody was at 1:10,000 dilution, and 
the GAPDH transfer membrane at a constant current $300 \mathrm{~mA}$ for $40 \mathrm{~min}$.

Luciferase reporter assay. The target genes of miR-217 were predicted using miRTarBase (http://mirtarbase.mbc. nctu.edu.tw/) and TargetScan (http://www.Targetscan.org/). The wild-type 3'UTR segment of the Tpd5212 mRNA (not the full length of Tpd5212 3'UTR) containing miR-217 binding sites was amplified and cloned into the Dual-luciferase reporter vector pGL3 (Promega, Madison, WI, USA) termed as: Wt-Tpd5212-3'UTR. A mutant construct in the miR-217 binding sites of the Tpd5212 3'UTR region also was generated by synthesis, and subcloned into the pGL3-control vector (Ambion, Foster City, CA, USA), and termed as Mut-Tpd52123'UTR. For the Dual-luciferase reporter assay, AsPC-1 cells were transfected with miR-217 or normal control for $24 \mathrm{~h}$, and then the cells were transfected with the Wt/Mut-Tpd52123'UTR reporter plasmid using Lipofectamine 2000. Forty-eight hours later, luciferase activity was measured using the Dual-Luciferase Reporter Assay kit (Promega) according to the manufacturer's instructions. Renilla luciferase was used for normalization.

Transwell invasion assay. Serum-starved AsPC-1 cells $\left(2 \times 10^{4}\right)$ were transferred to $8-\mu \mathrm{m}$ pore size cell culture inserts coated with $0.1 \%(\mathrm{w} / \mathrm{v})$ collagen. The cells were incubated in serumfree medium with or without thrombin/APC (both $10 \mathrm{nM}$ ), and the inserts were incubated at $37^{\circ} \mathrm{C}$ for $10 \mathrm{~h}$ in serum-free medium with MCP-1 (50 ng/ml) as a chemoattractant. For microscopic analysis, cells on the upper side of the Transwell membrane were removed with a cotton swab after which the inserts were fixed and stained in a crystal violet solution as previously described (22). The membranes were subsequently mounted on a glass slide, and migrated cells were counted by light microscopy. Cells were counted in 5 different fields using a magnification of $\mathrm{x} 20$.

Cell viability assay. Cell viability was determined using a Cell Counting Kit-8 (CCK-8; Beyotime) based on WST-8. WST-8 existing in CCK-8 solution reacts with mitochondrial dehydrogenase, and results in orange formazan deposition. A linear relationship between cell number and shade was used to evaluate cell proliferation. Briefly, AsPC- 1 cells $\left(2 \times 10^{3}\right)$ were seeded into 96-well plates in RPMI-1640 medium (100 $\mu \mathrm{l}$ ) containing 10\% FBS, and cultured overnight. After transfection for $4 \mathrm{~h}$, medium was renewed with fresh medium, and continuously cultured for $72 \mathrm{~h}$. CCK-8 solution $(10 \mu \mathrm{l})$ was added to each of the 96-well plates, and cultures were incubated for $90 \mathrm{~min}$ at $37^{\circ} \mathrm{C}$. Absorbance at $450 \mathrm{~nm}$ was measured using an automatic microplate reader (BioTeke, Beijing, China). A standard curve was constructed to deduce cell number. Experiments were performed in sextuplicate and repeated 3 times. Results were further analyzed using SPSS 20.0 software (SPSS, Inc., Chicago, IL, USA).

Cell cycle assay. Cell cycle distribution was examined using a Cell Cycle Analysis kit (MultiSciences, Susteren, The Netherlands). Cells were fixed with $70 \%$ ethanol at $4^{\circ} \mathrm{C}$ overnight, and treated with RNase A $(0.02 \mathrm{mg} / \mathrm{ml})$ in the dark at RT for $30 \mathrm{~min}$, and then stained with propidium iodide
(PI) and analyzed using a FACSCalibur flow cytometer (BD Biosciences, Franklin Lakes, NJ, USA) according to the manufacturer's instructions.

Wound scratch assay. Scratch assays were essentially performed as previously described (23). In detail, cells were seeded into 6-well plates in RPMI-1640 medium supplemented with $10 \%$ FBS. After the cells formed a confluent monolayer, a scratch was created in the center of the monolayer with a sterile p200 pipette tip. Next, the medium was removed and cells were washed with serum-free medium to remove floating debris. The cells were subsequently incubated for $18 \mathrm{~h}$ with serum-free medium with plasmids or control transfection. The ability of cells to close the wound was assessed by comparing the 0 and $24 \mathrm{~h}$ phase-contrast micrographs of 6 marked points along the wounded area. The percentage of non-recovered wound area was calculated by dividing the non-recovered area after $24 \mathrm{~h}$ by the initial area at $0 \mathrm{~h}$.

Statistical analysis. Data were analyzed using SPSS 20.0 software package (SPSS, Inc.) with independent samples t-test between two groups. All values were represented as mean \pm standard deviation (SD). Statistical significance was defined as $\mathrm{P}<0.05$.

\section{Results}

miR-217 expression is downregulated in HPAC tissues. First, we examined various miRNAs, including the difference in expression of miR-150, miR-138, miR-217, miR-205, miR-31 and miR-34, in HPAC and normal pancreas tissues. As shown in Fig. 1A, the expression of miR-150, miR-138 and miR-217 were downregulated in HPAC tissues and miR-217 showed the largest decrease when compared with the other miRNAs, while the expression of miR-205, miR-31 and miR-34 were increased. Thus, miR-217 was selected for subsequent study. Furthermore, to investigate the potential biological role of miR-217 expression in human HPAC progression, we evaluated miR-217 expression in 15 HPAC tissues and 15 normal pancreatic tissues by quantitative RT-PCR (qRT-PCR). As shown in Fig. 1B, the expression of miR-217 was significantly decreased in the HPAC tissues compared with that noted in the normal tissues.

Tpd52l2 is a target of miR-217 in HPAC cells. The target of miR-217 in HPAC cells was identified used bioinformatics software (miRTarBase and TargetScan). It was found that Tpd5212 3'UTR has a binding sequences for miR-217 at position 581-587 (Fig. 2A, upper panel). To further verify Tpd5212 as a direct target of miR-217, luciferase activity assay was performed. We found that miR-217 significantly inhibited the luciferase activity of 3'UTR of Tpd5212 in AsPC-1 cells (Fig. 2A, lower panel). Further experiments were performed to investigate the expression of Tpd5212 in 4 paired HPAC tissues and normal pancreas tissues. The results showed that Tpd5212 expression was upregulated in the HPAC tissues (Fig. 2C). The relationship between miR-217 and Tpd5212 expression in patients with HPAC was also investigated. Spearman's correlation analysis showed an inverse correlation between miR-217 expression 

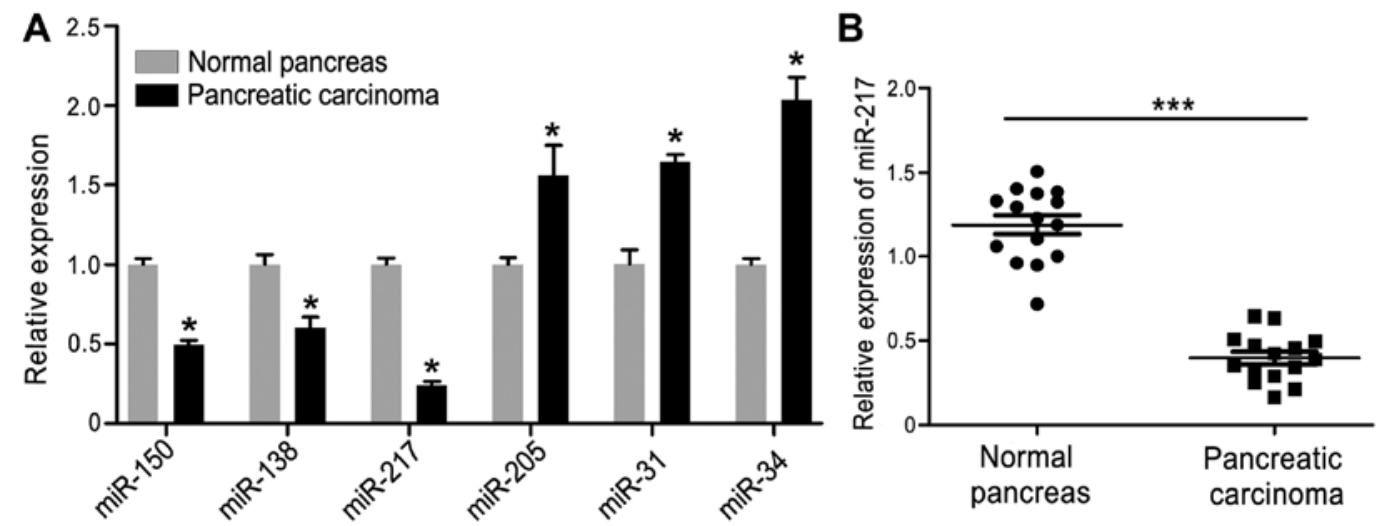

Figure 1. miR-217 expression is downregulated in pancreatic carcinoma samples. (A) Quantitative real-time polymerase chain reaction (qRT-PCR) analysis of miR-150, miR-138, miR-217, miR-205, miR-31 and miR-34 expression in pancreatic carcinoma tissues and normal pancreatic tissues; ${ }^{*} \mathrm{P}<0.05$ vs. normal pancreatic tissues. (B) qRT-PCR analysis of miR-217 expression in pancreatic carcinoma and normal pancreatic tissues. ${ }^{* * *} \mathrm{P}<0.001 \mathrm{vs}$. normal pancreatic tissues.

A

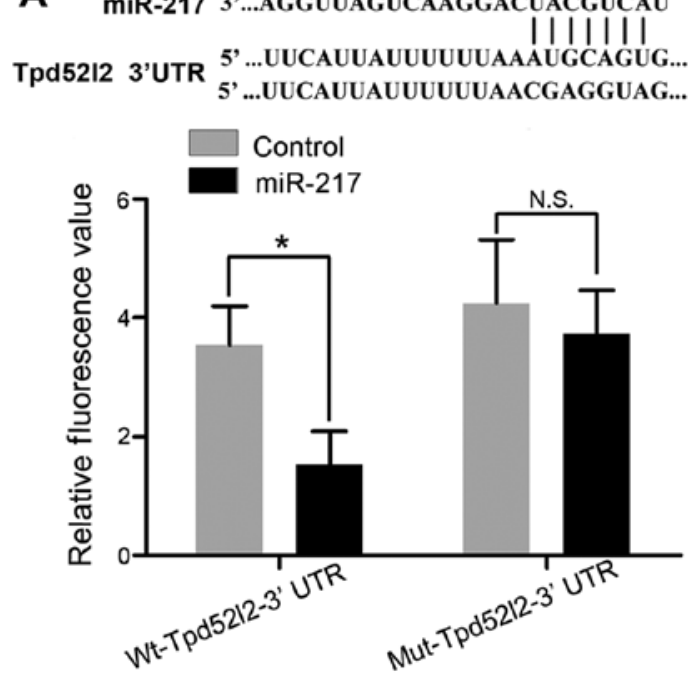

B
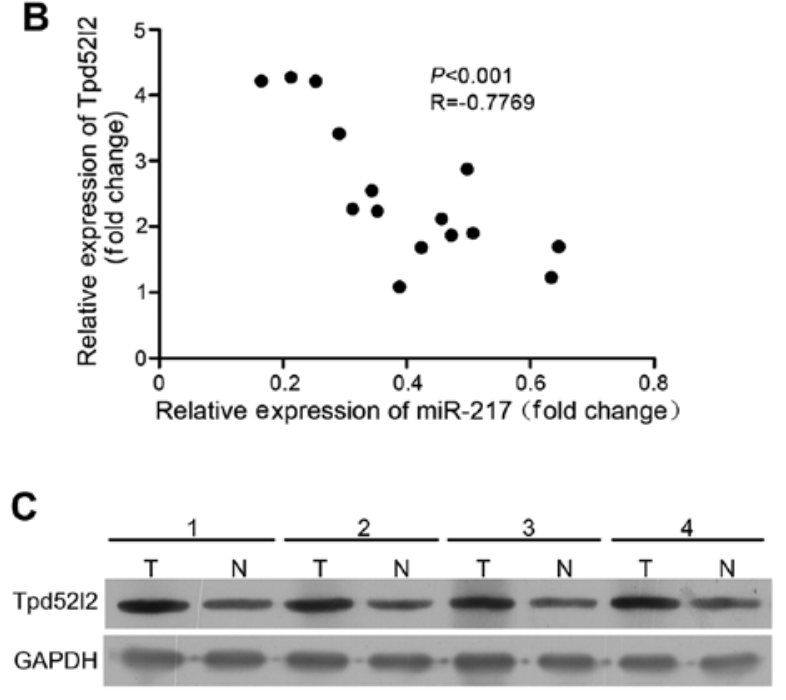

Figure 2. Tpd5212 is a target of miR-217 in HPAC cells. (A) Tpd5212 contains a binding sequence for miR-217 at position 581-587 (upper panel). The luciferase activity of AsPC-1 cells was determined after co-transfection with the miR-217 mimics or control and wild- or mutant-type report plasmid (lower panel); ${ }^{*} \mathrm{P}<0.05$ vs. related group; NS, no statistically significant difference. (B) Inverse relationship between Tpd5212 and miR-217 expression was demonstrated by Spearman's correlation in HPAC tissues. (C) Tpd5212 protein level was determined in 4 HPAC and normal tissues using western blotting. GAPDH was used as an internal control.

and Tpa5212 mRNA level in the HPAC tissues ( $\mathrm{r}=-0.7769$; $\mathrm{P}<0.001$ ) (Fig. 2B). These data suggest that Tpd5212 is a direct target of miR-217.

Downregulation of Tpd52l2 exhibits an effect similar to that of miR-217 overexpression in HPAC cells. To investigate the biological functions of Tpd5212 in HPAC cells, endogenous Tpd5212 was knocked down in AsPC-1 cells with specific siRNAs against Tpd5212 (si-Tpd5212). In addition, miR-217 mimics were transfected into AsPC-1 cells. We found that the mRNA level of miR-217 was increased by miR-217 mimic transfection, and Tpd5212 was significantly inhibited in AsPC-1 cells by si-Tpd5212 (Fig. 3A). Overexpression of miR-217 in AsPC-1 cells significantly inhibited cell proliferation (Fig. 3B), invasion and migration (Fig. 3D), induced apoptosis (Fig. 3E) and caused cell cycle arrest at the G0/G1 phase (Fig. 3F). Similarly, downregulation of Tpd5212 in AsPC-1 cells significantly inhibited cell proliferation (Fig. 3C), invasion and migration (Fig. 3D), induced apoptosis (Fig. 3E) and cell cycle arrest (Fig. 3F). These results suggest that silencing of Tpd5212 had a similar effect as miR-217 overexpression in the HPAC cells.

Overexpression of Tpd52l2 reverses the effects of miR-217 in HPAC cells. To investigate the functional relevance of the targeting of Tpd5212 by miR-217, we assessed whether Tpd5212 overexpression reverses the inhibitory effects of miR-217 restoration on AsPC-1 cell proliferation, migration, invasion, apoptosis and cell cycle distribution. AsPC-1 cells were co-transfected with miR-217 or control mimics and Tpd5212 overexpression plasmids. qRT-PCR analysis was used to validate the Tpd5212 mRNA level in the rescue experiment (Fig. 4A). In addition, our results also showed that the exogenous expression of Tpd5212 reversed the effects of miR-217 

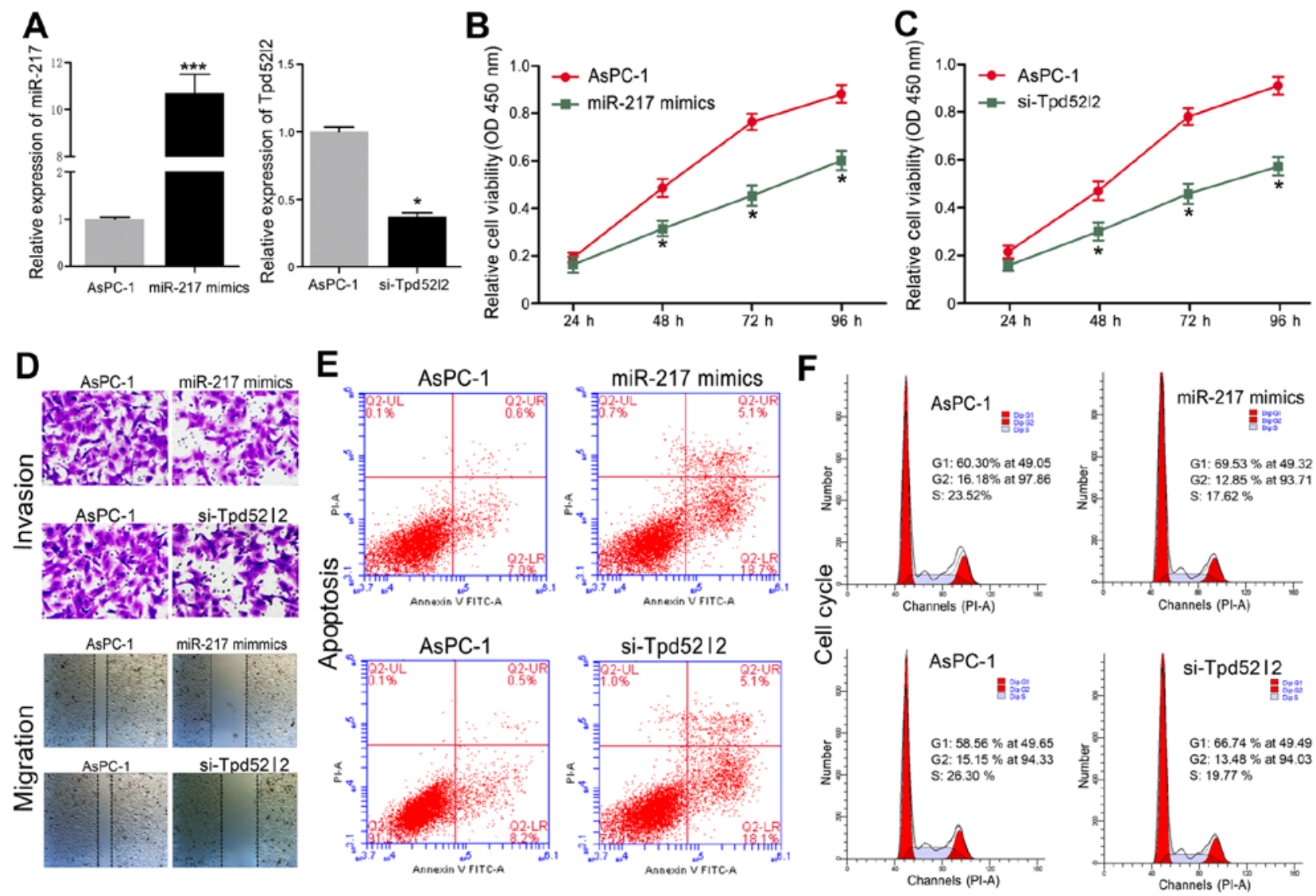

Figure 3. miR-217 inhibits cell proliferation, migration and invasion and induces apoptosis and cell cycle arrest in human HPAC cells. Downregulation of Tpd5212 has effects similar to those following overexpression of miR-217 in HPAC cells. (A) The expression levels of miR-217 and Tpd5212 were detected in miR-217 mimic- and Tpd5212 siRNA-transfected cells by qRT-PCR; ${ }^{*} \mathrm{P}<0.05,{ }^{* * *} \mathrm{P}<0.001$ vs. control. (B and C) Cell proliferation was determined in AsPC-1 cells transfected with miR-217 mimics or Tpd5212 siRNA; ${ }^{2} \mathrm{P}<0.05$ vs. control. (D and E) Migration, invasion and apoptosis were determined in AsPC-1 cells transfected with miR-217 mimics or Tpd5212 siRNA. (F) Cell cycle distribution was analyzed in AsPC-1 cells transfected with miR-217 mimics or Tpd5212 siRNA. Both overexpression of miR-217 and downregulation of Tpd5212 arrested the cell cycle of the HPAC cells at the G0/G1 phase.
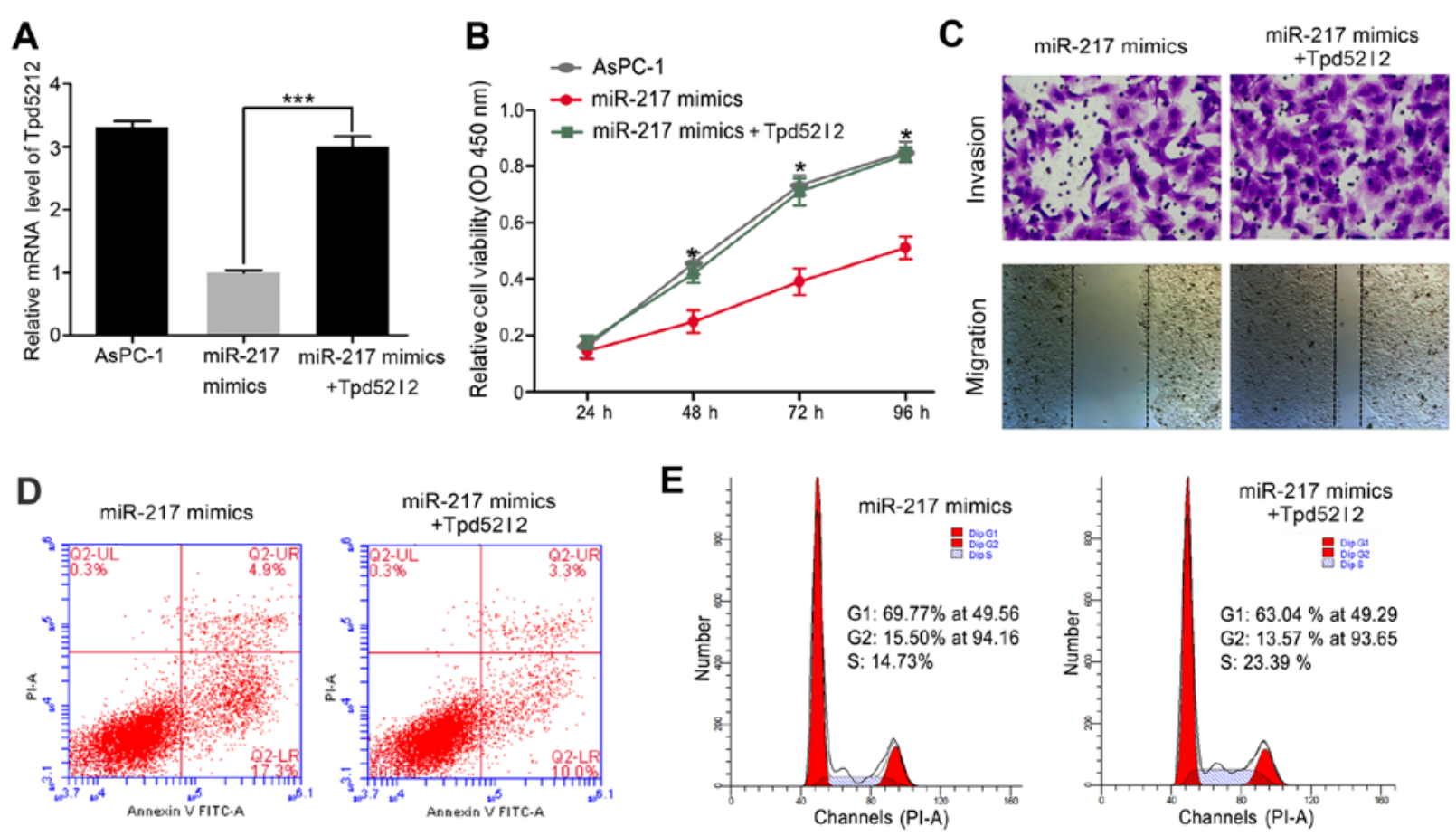

Figure 4. Overexpression of Tpd5212 reverses the effects of miR-217 in HPAC cells. (A) Tpd5212 expression at the mRNA level in AsPC-1 cells co-transfected with Tpd5212 overexpression plasmid and miR-217 mimic or control by qRT-PCR; ${ }^{* * *} \mathrm{P}<0.001$ vs. related group (B-E) Cell proliferation, migration, invasion, apoptosis and cell cycle distribution were determined in AsPC-1 cells transfected with miR-217 mimic with/without Tpd5212 overexpression plasmid. Upregulation of Tpd5212 reversed the effects of the overexpression of miR-217 in the AsPC-1 cells; ${ }^{*} \mathrm{P}<0.05$ vs. miR-217. 


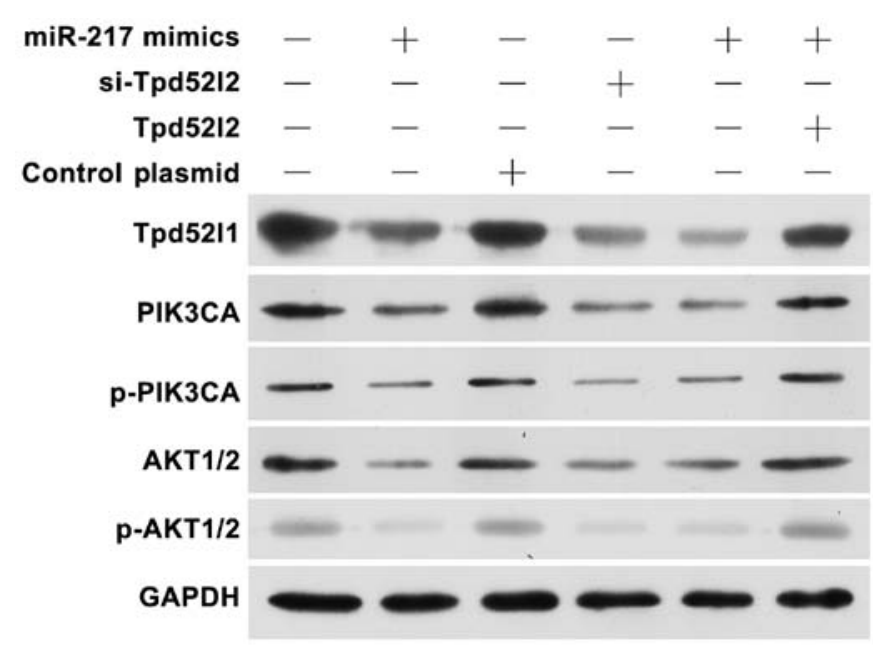

Figure 5. Overexpression of miR-217 or knockdown of Tpd5212 inhibits the PIK3CA/AKT signaling pathways. AsPC-1 cells were transfected with miR-217 mimics, si-Tpd5212 or Tpd5212 plasmids. After $24 \mathrm{~h}$, cells were subjected to western blot analysis.

overexpression on cell proliferation, migration, invasion, apoptosis and cell cycle distribution (Fig. 4B-E).

Overexpression of miR-217 or knockdown of Tpd52l2 inhibits the PIK3CA/AKT signaling pathways. To further explain the effect of miR-217/Tpd5212 on HPAC cells, we detected the PIK3CA/AKT signaling pathways in miR-217 mimic, si-Tpb5212 or Tpd5212 overexpression plasmid transfected cells (Fig. 5). The results showed that overexpression of miR-217 or knockdown of Tpd5212 suppressed the PIK3CA/pPIK3CA and AKT1/2/p-AKT1/2 protein levels. In addition, following co-transfection of miR-217 and the Tpd5212 plasmid, the inhibitory effect of miR-217 was reversed. All these data indicated that upregulation of miR-217 exerts an inhibitory effect on HPAC growth and metastasis partially by suppressing Tpd5212 expression.

\section{Discussion}

Evidence shows that miRNAs are involved in tumorigenesis and metastasis of various types of human cancers, including HPAC $(15,24,25)$. Aberrant expression of miR-217 has been found in various human cancers (11-17). Frequently, miR-217 expression is downregulated and acts as a tumor suppressor, while it is overexpressed and functions as an oncogene in breast cancer (17) and B cell lymphoma (26). However, the function and relevant mechanisms of miR-217 in HPAC have not been comprehensively identified.

In the present study, we found that the expression of miR-217 was significantly downregulated in HPAC tissues and cells. We also demonstrated that restoration of miR-217 expression in HPAC cells inhibited proliferation, migration, and invasion, induced apoptosis and caused cell cycle arrest. These results suggested that miR-217 functions as a tumor suppressor in HPAC.

To further investigate the molecular mechanism of the tumor-suppressor role of miR-217 in HPAC, we used bioinformatics software to identify the target of miR-217 in HPAC cells. Tpd5212 3'UTR was found to have a binding sequence for miR-217 at position 581-587. Luciferase activity assay, qRT-PCR and western blot assay further confirmed that Tpd5212 is a target gene of miR-217. Tpd5212 is a member of the TPD52 family which has been implicated in multiple human cancers (18-20). Studies have demonstrated that TPD52 may be important for maintaining tumorigenesis and metastasis of cancer cells (21). In the present study, we found that Tpd5212 expression was upregulated and inversely correlated with miR-217 expression in HPAC tissues. In addition, we also found that downregulation of Tpd5212 had a similar effect as the restoration of miR-217 expression in HPAC cells, and overexpression of Tpd5212 reversed the effects of miR-217 in AsPC-1 cells. These results suggest that miR-217 exerts a tumor-suppressor role in HPAC, at least in part, by suppressing Tpd5212 expression.

In summary, the present study demonstrated that miR-217 expression is downregulated in HPAC cells and tissues, and that restoration of miR-217 expression inhibited proliferation, migration and invasion, induced apoptosis and cell cycle arrest of HPAC cells by suppressing Tpd5212. PIK3CA/ AKT signaling pathways generally act to promote survival through inhibition of pro-apoptotic factors and activation of anti-apoptotic factors (27). Activation of the PIK3CA/AKT pathway signals through mTOR to promote protein translation and cell cycling, and other effectors participate in regulation of transcription, apoptosis and cellular metabolism (28-30). In addition, PIK3A, at the top of the pathway, is an upstream catalytic enzyme that, when active, leads to cell growth and proliferation and in particular inhibition of cell death (31). Finally, we found that overexpression of miR-217 or knockdown of Tpd5212 inhibited the PIK3CA/AKT signaling pathways. In addition, this may explain the effect of miR-217/ Tpd5212 on HPAC development. Taken together, targeting of the miR-217/Tpd5212 axis may be a new therapeutic strategy by which to treat patients with HPAC.

\section{Acknowledgements}

The present study was supported by grants from the National Nature Science Foundation of China (nos. 81672449 and 81272239). The present study was also supported by Talents Planning of Six Summit Fields of Jiangsu Province (WSN-025).

\section{References}

1. Siegel RL, Miller KD and Jemal A: Cancer statistics, 2015. CA Cancer J Clin 65: 5-29, 2015.

2. Ryan DP, Hong TS and Bardeesy N: Pancreatic adenocarcinoma. N Engl J Med 371: 1039-1049, 2014.

3. Valinezhad Orang A, Safaralizadeh R and KazemzadehBavili M: Mechanisms of miRNA-mediated gene regulation from common downregulation to mRNA-specific upregulation. Int J Genomics 2014: 970607, 2014.

4. Bushati N and Cohen SM: microRNA functions. Annu Rev Cell Dev Biol 23: 175-205, 2007.

5. Hwang HW and Mendell JT: MicroRNAs in cell proliferation, cell death, and tumorigenesis. Br J Cancer (Suppl 96): R40-R44, 2007.

6. Kwak PB, Iwasaki S and Tomari Y: The microRNA pathway and cancer. Cancer Sci 101: 2309-2315, 2010.

7. Zhang S, Liu X, Liu J, Guo H, Xu H and Zhang G: PGC-1 alpha interacts with microRNA-217 to functionally regulate breast cancer cell proliferation. Biomed Pharmacother 85: 541-548, 2017.

8. Yates LA, Norbury CJ and Gilbert RJ: The long and short of microRNA. Cell 153: 516-519, 2013. 
9. Cioffi M, Trabulo SM, Sanchez-Ripoll Y, Miranda-Lorenzo I, Lonardo E, Dorado J, Reis Vieira C, Ramirez JC, Hidalgo M, Aicher A, et al: The miR-17-92 cluster counteracts quiescence and chemoresistance in a distinct subpopulation of pancreatic cancer stem cells. Gut 64: 1936-1948, 2015.

10. Neesse A and Gress TM: Emerging role of microRNAs to tackle drug resistance in pancreatic cancer. Gut 64: 1842-1843, 2015.

11. Li J, Li D and Zhang W: Tumor suppressor role of miR-217 in human epithelial ovarian cancer by targeting IGF1R. Oncol Rep 35: 1671-1679, 2016.

12. Wei R, Deng Z and Su J: miR-217 targeting Wnt5a in osteosarcoma functions as a potential tumor suppressor. Biomed Pharmacother 72: 158-164, 2015.

13. Su J, Wang Q, Liu Y and Zhong M: miR-217 inhibits invasion of hepatocellular carcinoma cells through direct suppression of E2F3. Mol Cell Biochem 392: 289-296, 2014.

14. Zhao WG, Yu SN, Lu ZH, Ma YH, Gu YM and Chen J: The miR-217 microRNA functions as a potential tumor suppressor in pancreatic ductal adenocarcinoma by targeting KRAS. Carcinogenesis 31: 1726-1733, 2010.

15. Guo J, Feng Z, Huang Z, Wang H and Lu W: MicroRNA-217 functions as a tumour suppressor gene and correlates with cell resistance to cisplatin in lung cancer. Mol Cells 37: 664-671, 2014.

16. Wang B, Shen ZL, Jiang KW, Zhao G, Wang CY, Yan YC, Yang Y, Zhang JZ, Shen C, Gao ZD, et al: MicroRNA-217 functions as a prognosis predictor and inhibits colorectal cancer cell proliferation and invasion via an AEG-1 dependent mechanism. BMC Cancer 15: 437, 2015.

17. Zhang Q, Yuan Y, Cui J, Xiao T and Jiang D: miR-217 promotes tumor proliferation in breast cancer via targeting DACH1. J Cancer 6: 184-191, 2015.

18. Byrne JA, Balleine RL, Schoenberg Fejzo M, Mercieca J, Chiew YE, Livnat Y, St Heaps L, Peters GB, Byth K, Karlan BY, et al: Tumor protein D52 (TPD52) is overexpressed and a gene amplification target in ovarian cancer. Int J Cancer 117: 1049-1054, 2005.

19. Rubin MA, Varambally S, Beroukhim R, Tomlins SA, Rhodes DR, Paris PL, Hofer MD, Storz-Schweizer M, Kuefer R, Fletcher JA, et al: Overexpression, amplification, and androgen regulation of TPD52 in prostate cancer. Cancer Res 64: 3814-3822, 2004.
20. Zhu H, Lam DC, Han KC, Tin VP, Suen WS, Wang E, Lam WK, Cai WW, Chung LP and Wong MP: High resolution analysis of genomic aberrations by metaphase and array comparative genomic hybridization identifies candidate tumour genes in lung cancer cell lines. Cancer Lett 245: 303-314, 2007.

21. Byrne JA, Mattei MG and Basset P: Definition of the tumor protein D52 (TPD52) gene family through cloning of D52 homologues in human $(h D 53)$ and mouse $(m D 52)$. Genomics 35 : 523-532, 1996

22. Queiroz KC, Shi K, Duitman J, Aberson HL, Wilmink JW, van Noesel CJ, Richel DJ and Spek CA: Protease-activated receptor-1 drives pancreatic cancer progression and chemoresistance. Int J Cancer 135: 2294-2304, 2014

23. Lin C, von der Thüsen J, Daalhuisen J, ten Brink M, Crestani B, van der Poll T, Borensztajn K and Spek CA: Pharmacological targeting of protease-activated receptor 2 affords protection from bleomycin-induced pulmonary fibrosis. Mol Med 21: 576-583, 2015.

24. Chang W, Liu M, Xu J, Fu H, Zhou B, Yuan T and Chen P: MiR-377 inhibits the proliferation of pancreatic cancer by targeting Pim-3. Tumour Biol 37: 14813-14824, 2016.

25. Yonemori K, Kurahara H, Maemura $K$ and Natsugoe $S$ : MicroRNA in pancreatic cancer. J Hum Genet 62: 33-40, 2017.

26. Slattery ML, Lundgreen A, Herrick JS, Caan BJ, Potter JD and Wolff RK: Associations between genetic variation in RUNX1, RUNX2, RUNX3, MAPK1 and eIF4E and risk of colon and rectal cancer: Additional support for a TGF- $\beta$-signaling pathway. Carcinogenesis 32: 318-326, 2011.

27. Nathan N, Keppler-Noreuil KM, Biesecker LG, Moss J and Darling TN: Mosaic disorders of the PI3K/PTEN/AKT/TSC/ mTORC1 signaling pathway. Dermatol Clin 35: 51-60, 2017.

28. Samuels Y and Velculescu VE: Oncogenic mutations of PIK3CA in human cancers. Cell Cycle 3: 1221-1224, 2004.

29. Hennessy BT, Smith DL, Ram PT, Lu Y and Mills GB: Exploiting the PI3K/AKT pathway for cancer drug discovery. Nat Rev Drug Discov 4: 988-1004, 2005.

30. Zhao L and Vogt PK: Class I PI3K in oncogenic cellular transformation. Oncogene 27: 5486-5496, 2008.

31. Fleisher B, Clarke C and Ait-Oudhia S: Current advances in biomarkers for targeted therapy in triple-negative breast cancer. Breast Cancer 8: 183-197, 2016. 